\title{
Sexuality of Moroccan Survivors of Cervical Cancer: A Prospective Data
}

\author{
Zineb Dahbi*, Ali Sbai, Loubna Mezouar
}

\begin{abstract}
Introduction: This is a prospective study aiming to describe how cervical cancer and its treatment can affect the sexuality of a specific population of Moroccan women survivors of this disease. Materiels and Methods: It is a comparison of clinical features and Female Sexual Function Index (FSFI) of 3 balanced groups of women, made up of 100 survivors of cervical cancer, 100 survivors of non-gynecological cancer and a group of 100 healthy women. Results: $97 \%$ of the patients stopped their full sexual activity at the time of treatment; the time interval between treatment and regular sexual activity was 8 months for the cervical cancer group, and 5.8 months for non-gynecologic cancer $(\mathrm{P}$ $=0.001$ ). Vaginal length assessed by pelvic examination during follow up visits was estimated at about $6.2 \mathrm{~cm}, 9.2 \mathrm{~cm}$ and $9.5 \mathrm{~cm}$ respectively for the cervical cancer group, non-gynecologic cancer group and the control group ( $\mathrm{p}=0.04)$. On the basis of the FSFI questionnaire, the analysis of the 6 main sexuality parameter scores did attest worse results for the variables related to sexual function for cervical cancer group, but not in a statistically significant way. Conclusion: In order to preserve an optimal quality of life after cervical cancer, the sexual dimension is a crucial parameter to take into consideration throughout treatment phases, especially radiation (by encouraging sexual intercourses, and using vaginal dilators during the treatment). Moroccan survivors of this disease must be treated according to a multidisciplinary approach, that includs the psychological component.
\end{abstract}

Keywords: Cervical cancer- cancer survivors- Moroccan women- sexuality

Asian Pac J Cancer Prev, 19 (11), 3077-3079

\section{Introduction}

Cervical cancer is the second most common female cancer worldwide, according to 2005 statistics of the Moroccan cancer statistical record. The national cancer society estimated that 6,500 new cases of invasive cervical cancer are diagnosed annually. Its incidence rate increased by 1.9 per 100,000 compared to 2004 , despite education campaigns and the mass adhesion to the screening protocol of cervical cancer. The management of the disease depends on the diagnosis of the tumor stage, which may include a multimodal treatment (surgery, radiation, chemo radiation, and chemotherapy for palliative purposes). Prognosis of this disease has been upstaged nowadays by the available treatments and screening protocols.

Therefore, the quality of life worldwide has become the main aim of cervical cancer management programs. Curiously, despite the fact that sexuality is a capital dimension of the quality of life, it doesn't get enough focus in the Moroccan practice. Actually, there are few studies that examine the sexuality of Arab survivors of cervical cancer, and how it is affected by the after-effects of the disease, and the late treatment toxicities.
The aim of this study is to describe how cervical cancer and its treatment affect the sexuality of women survivors of this disease.

\section{Materials and Methods}

This is a prospective study, conducted from September 2014 to June 2015; in the university hospital center of Oujda, Morocco, including 3 balanced groups of Moroccan women: the first group included 100 survivors of cervical cancer, the second group 100 survivors of a non-gynecologic cancer, and the third group was made up of 100 healthy Moroccan women. The first two groups of cancer survivors were randomly selected from the hospital archives data from their checkup appointments. They willingly agreed to be part of this study. The women in the third group were randomly picked up from hospital visitors volunteering to respond to the questionnaire.

There was no age limit to take part in the study. The same doctor performed the questioning, and patients with a poor command of the Arabic language were excluded.

The three groups were asked to answer a questionnaire about their actual sexual life, adapted from the Female

Department of Radiation Oncology, University Hospital Mohammed VI, Oujda, Morocco. *For Correspondence: Beniz899@hotmail.com 
Sexual Function Index (FSFI). They were asked to define the importance of desire, the frequency of their sexual activity and the level of satisfaction or frustration they felt about it.

The primary end point was sexual dysfunction incidence; the second end point was correlation between cervical cancer treatment and sexual dysfunction.

\section{Results}

The mean age of the participants was 52.8 years, the epidemiologic data of the 3 groups did not show any statistically significant difference, in terms of age at treatment or at the study, and also in terms of the marital status and the level of education (Table1).

For the cervical cancer group, 34\% of patients had received pelvic surgery, $95 \%$ underwent pelvis radiation and $67 \%$ had chemotherapy. For the non gynecologic cancer patients, none of them had pelvic surgery or pelvic radiation, while $95 \%$ of them had chemotherapy. Relating to the after treatment sexual life, only $12 \%$ of the cervical cancer group reported receiving guidance in this matter. Parameters that can be associated with the adverse effects of treatment were different between the two cancer groups $(\mathrm{p}=0.03)$, with the greater frequency occurring in the cervical cancer group: bleeding during or after sexual relations, incontinence and retention of urination; increase of diarrhea; anal pain; intestinal bleeding and lymphedema in lower limbs. Vaginal length assessed by pelvic examination during follow-up visits was estimated to be at about $6.2 \mathrm{~cm}, 9.2 \mathrm{~cm}$ and $9.5 \mathrm{~cm}$ respectively for

Table 1. Epidemiological and Clinical Features of the $\underline{\text { Patients }}$

\begin{tabular}{|c|c|c|c|}
\hline & $\begin{array}{l}\text { Cervix } \\
\text { cancer } \\
\text { group }\end{array}$ & $\begin{array}{l}\text { Non gynecologic } \\
\text { cancer group }\end{array}$ & $\begin{array}{l}\text { Control } \\
\text { group }\end{array}$ \\
\hline \multicolumn{4}{|l|}{ Age (Mean) } \\
\hline At treatment & 45.2 & 53.6 & - \\
\hline At the study & 51.3 & 55.2 & 49.2 \\
\hline \multicolumn{4}{|l|}{ Menopause status (\%) } \\
\hline Yes & 33.5 & 43.7 & 31.3 \\
\hline No & 66.5 & 56.3 & 68.7 \\
\hline \multicolumn{4}{|l|}{ Marital status (\%) } \\
\hline Single & 17.3 & 18.3 & 14.0 \\
\hline Married & 26.4 & 39.7 & 54.1 \\
\hline Divorced - widowed & 56.3 & 42.0 & 31.9 \\
\hline \multicolumn{4}{|l|}{ Level of education } \\
\hline None & 29.3 & 18.8 & 19.0 \\
\hline Primary & 33.2 & 29.6 & 26.9 \\
\hline Secondary - universetary & 37.5 & 51.6 & 54.3 \\
\hline \multicolumn{4}{|l|}{ Employment (\%) } \\
\hline Yes & 23.0 & 36.0 & 38.0 \\
\hline No & 77.0 & 64.0 & 62.0 \\
\hline \multicolumn{4}{|l|}{ Treatment (\%) } \\
\hline Pelvic surgery & 34.0 & 0.0 & 5 \\
\hline Pelvic radiation & 95.0 & 0.0 & - \\
\hline chemotherapy & 67.0 & 95.0 & - \\
\hline
\end{tabular}

Table 2. FSFI Scores Parameters

\begin{tabular}{lccc}
\hline $\begin{array}{l}\text { FSFI domain } \\
\text { score }\end{array}$ & $\begin{array}{c}\text { Cervix } \\
\text { cancer } \\
\text { group }\end{array}$ & $\begin{array}{c}\text { Non gynecologic } \\
\text { cancer group }\end{array}$ & Control group \\
\hline Desire & $25+28$ & $28+11$ & $39+16$ \\
Arouasal & $29+13$ & $31+2$ & $38+21$ \\
Lubrification & $36+15$ & $31+18$ & $58+2$ \\
Orgasm & $33+05$ & $33+14$ & $45+1$ \\
Satisfaction & $37+18$ & $38+22$ & $31+13$ \\
Pain & $39+12$ & $39+11$ & $32+05$ \\
Total & $199+32$ & $\mathrm{P}=02320+25$ & $\mathrm{P}=06724+32$ \\
\hline
\end{tabular}

the cervical cancer group, the non gynecologic cancer group and the control group ( $\mathrm{p}=0.04) .97 \%$ of patients stopped their full sexual activity at treatment; the time interval between treatment and regular sexual activity was 8 months for the cervical cancer group, and 5.8 months for the non-gynecologic cancer group $(\mathrm{P}=0.001)$. On the basis of the FSFI questionnaire, the analysis of the 6 main sexuality parameters scores did attest worse results for the variables related to sexual function for the cervical cancer group (Table 2), but there was no significant difference between them and the control group; or between the cervical cancer and non-gynecologic cancer group. In fact, the total FSFI score mean was 19.9 in the cervix cancer group, classified as sexual dysfunction because the cutoff point is situated below 26 (Table 2).

\section{Discussion}

It is well known that sexual dysfunction may occur after pelvic surgery and radiation as treatments of cervical cancer (Maher and Denton, 2008; Andersen et al., 1997) It results in vaginal atrophy, vaginal dryness, dyspareunia, and frequent urinary tract infections. Hysterectomy and abdominal scarring may distort a woman's body image and be sexual disorder determining factors (Donovan et al., 2007; Golbasi and Erenel, 2012). Chemotherapy also influences the patient's general condition by increasing fatigue. Psychological factors such as depression and anxiety can also contribute to sexual problems by decreasing sexual desire. However, those factors are not exclusive of cervical cancer, (Carter et al., 2012; Cleary and Hegarty, 2001; Herzog and Wright, 2007). Actually, our study shows that sexual disorders are not a sine qua non result of cervical cancer or its treatment for patients who did not show evidence of the disease after primary treatment, compared to non-gynecologic cancers, and with healthy women.

So far, there has been no published study with a similar design. In addition, little data regarding cancer survivor sexuality in Morocco is available. For breast cancer survivors, Sbittii et al., (2011) found that 78\% of the patients had never before suffered from sexual dysfunctions; $19 \%$ of the dysfunctions were observed after chemotherapy, 9\% after surgery and 3\% after radiotherapy. The totality (100\%) expressed the view that they had not received enough information about how the disease and treatment (including surgery) might affect their sexual life. About this specific point, Errihani et al., 
(2010) have reported a lack of communication as being one of the main problems in recognizing and handling sexual problems in the Moroccan population. Often out of morality reasons and questions of decency, patients feel embarrassed to speak about their sexuality. Doctors do not take the initiative of asking questions about sexuality as long as their patients remain silent about it; they wait until the patients put the questions to them. Quite often, they incorrectly deduce that because their patients are not talking about it, they are satisfied with their sexuality. However, in order to compare our results with other studies on sexuality of foreign cancer survivors, we may here refer to a Japanese study on this matter, published in September 2014, (Ratner et al., 2010). It showed that quality of life and sexual function in cervical cancer survivors were compromised compared to those of the general population. In addition, pelvic radiation was found to be associated with poor quality of life and sexual function. (White, 2008; Baser et al., 2012). Camila Soares, Lima Corrend et al found that for cervical cancer survivors, sexual dysfunction was higher compared to the control group, leading to think that cervical cancer has a negative effect on the sexuality of the subjects studied (Corrêa et al., 2016).

There is little data regarding cancer survivors sexuality in Morocco, . For breast cancer survivors, Sbittti et al., (2011) found that $78 \%$ of them had no sexual dysfunctions prior to the cancer diagnosis. Furthermore, they found that chemotherapy was associated with $19 \%$ of the observed dysfunctions, $9 \%$ of the dysfunctions was due to surgery, and $3 \%$ to radiotherapy. None of the interviewed patients received information about how breast cancer or its treatments might affect their sexuality (Yassir et al., 2011). About that, Errihani et al., (2010) have argued that a lack of communication was one of the main problems in admitting and treating sexual problems among the Moroccan population. For questions of morality and perceived decency, Moroccan women generally avoid talking about their sexuality, even to their doctors. In terms of management of sexual disorders shown by the women in our study, and although the level of sexual dysfunction was quite similar between the two studied groups, physicians should be more alert to the need to provide targeted assistance to their patients during treatment time and follow up monitoring. For example, vaginal dryness was posed a real problem for cervical cancer survivors, this symptom may be improved by applying vaginal lubricants and/or local estrogen cream.

In conclusion, we fell short of showing an additional impact of cervical cancer or its treatment on the sexuality of the survivors, since the women in the 3 groups presented some sexual dysfunctions. However, sexuality remains an important parameter of quality of life, and it should be taken into consideration during the treatment and after it.

\section{Ethics approval and consent to participate}

All procedures performed in this study involving human participants were in accordance with the ethical standards of the National Research Committee.

\section{Consent for publication}

All participants approved the publication of this data.

\section{Availability of data and material}

All data generated or analyzed during this study are included in this published article.

\section{Funding}

No funding was needed to accomplish this study.

\section{Authors' contributions}

All the authors contributed to the article and approved the final version of the manuscript.

\section{Conflicts of interests}

The authors declare that they have no competing interests.

\section{References}

Andersen BL, Woods XA, Copeland LJ (1997). Sexual self-schema and sexual morbidity among gynecologic cancer survivors. J Consult Clin Psychol, 65, 221-9.

Baser RE, Li Y, Carter J (2012). Psychometric validation of the female sexual function index (FSFI) in cancer survivors. Cancer, 118, 4606-18.

Corrêa CS, Leite IC, Andrade AP, et al (2016). Sexual function of women surviving cervical cancer. Arch Gynecol Obstet, 293, 1053-63.

Carter J, Penson R, Barakat R, Wenzel L (2012). Contemporary quality of life issues affecting gynecologic cancer survivors. Hematol Oncol Clin North Am, 26, 169-94.

Cleary V, Hegarty J (2011). Understanding sexuality in women with gynaecological cancer. Eur J Oncol Nurs, 15, 38-45.

Donovan KA, Taliaferro LA, Alvarez EM, et al (2007). Sexual health in women treated for cervical cancer: characteristics and correlates. Gynecol Oncol, 104, 428-34.

Errihani H (2010). Impact of cancer on sexuality: How is the Moroccan patient affected?. Sexologies, 19, 92-8.

Golbasi Z, Erenel AS (2012). The quality of sexual life in womenwith gynaecological cancers . Arch Gynecol Obstet, 285, 1713-7.

Herzog TJ, Wright JD (2007). The impact of cervical cancer on quality of life-the components and means for management. Gynecol Oncol, 107, 572-7.

Maher EJ, Denton A (2008). Survivorship, late effects and cancer of the cervix. Clin Oncol, 20, 479-87.

Ratner ES, Foran KA, Schwartz PE, Minkin MJ (2010). Sexuality and intimacy after gynecological cancer. Maturitas, 66, 23-6.

White ID (2008). The assessment and management of sexual difficulties after treatment of cervical and endometrial malignancies. Clin Oncol, 20, 488-96.

Yassir S, Habiba K, Ismail E (2011). Breast cancer treatment and sexual dysfunction: Moroccan women's perception. $B M C$ Womens Health, 11, 29.

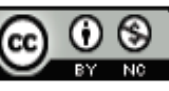

This work is licensed under a Creative Commons AttributionNon Commercial 4.0 International License. 